\title{
Similar dark matter and baryon abundances with TeV-scale leptogenesis
}

\author{
Sacha Davidson and Martin Elmer \\ IPNL, Université de Lyon, Université Lyon 1, \\ CNRS/IN2P3, 4 rue E. Fermi 69622 Villeurbanne cedex, France \\ E-mail: m.elmer@ipnl.in2p3.fr, s.davidson@ipnl.in2p3.fr
}

ABStract: We estimate the Baryon Asymmetry of the Universe (BAU) produced in an inverse seesaw model containing extra light singlets, and with lepton number conservation prior to the electroweak phase transition. A CP asymmetry $\epsilon \sim \mathcal{O}(1)$ is required to obtain a large enough BAU. We discuss the relation between the baryon and WIMP relic densities in baryogenesis scenarios using the out-of-equilibrium decay of a baryon-parent of mass $M$ : when baryon number violation freezes out, the remaining density of baryon-parents is $\sim M / m_{W} \times$ the WIMP relic density. So the baryon/WIMP ratio is $\sim \epsilon M / m_{W}$. A natural explanation of the similar WIMP and baryon densities could be that CP violation is of order the ratio $m_{W} / M$.

Keywords: Cosmology of Theories beyond the SM, Neutrino Physics

ARXIV EPRINT: 1208.0551 


\section{Contents}

1 Introduction 1

2 Review, notation and masses 2

2.1 Observations 2

2.2 Models 3

2.3 Notation and masses 5

3 Thermal history $\quad 7$

$3.1 \psi \bar{\psi} \rightarrow \phi \bar{\phi}$ annihilations $\quad 7$

3.2 Decays and the CP asymmetry 8

$\begin{array}{ll}\text { 3.3 Washout of the asymmetry by inverse decays and scattering } & 10\end{array}$

3.4 Putting it all together in the Majorana case 11

$\begin{array}{ll}3.5 & \text { Dirac limit } \\ & 12\end{array}$

$\begin{array}{lll}4 & \text { Discussion } & 13\end{array}$

\section{Introduction}

In recent years, there has been some interest in relating the cosmological number density of baryons [1-4], with the number density of dark matter particles (for a review of cold dark matter, see $[5,6])$. A popular approach, refered to as Asymmetric Dark Matter (ADM) [7-19], is to implement a single mechanism that generates an excess of particles over anti-particles, which materialises both in the baryons and in the dark matter. However, it is not necessary to relate the amounts of Dark Matter and baryons, but rather, to explain that both have their observed values. This (subtle) distinction is relevant, because the relic density of a weakly interacting particle (WIMP) is naturally of order the observed dark matter density. So it is not clear what is gained by dropping the "WIMP miracle", to link the dark matter number density to the baryon asymmetry, which depends on an arbitrary $\mathrm{CP}$ violation parameter. Scenarios which implement the link in the inverse direction [2023], explaining the baryon number density from the dark matter density, could be more interesting. But they require a $\mathrm{CP}$ asymmetry that is naturally $\mathcal{O}(1)$. The aim of this paper is to follow the "subtle" distinction mentioned above: we assume that the Dark Matter are WIMPs (so their observed relic abundance is already natural and requires no explanation), and attempt to build a baryogenesis model, which naturally generates, in a similar cosmology, the observed baryon asymmetry.

We consider an "inverse seesaw"-like model [24], extending the Standard Model(SM) with TeV-scale electroweak singlet Dirac neutrinos $\psi$, additional light singlet fermions $s$ and a $L=2$ singlet scalar $\phi$. The $\psi$ participate in Yukawa interactions with SM neutrinos, and 
the $\phi$ have ( $L$ conserving) interactactions with $\psi$ and $s$. For generic choices of couplings, lepton number violation arises spontaneously when $\phi$ gets a vev, after the electroweak phase transition, giving Majorana masses to the doublet SM neutrinos. However, there is a particular limit where lepton number is conserved and the SM neutrinos are Dirac, whose baryogenesis prospects are explored in section 3.5. Section 2 reviews our model and notation. Since we are building a model, we would like it to exhibit as many interesting features as possible. Our model can fit the observed neutrino mass differences. However, the additional light singlets cannot fit the reactor neutrino anomaly [25-27] and Big Bang Nucleosynthesis; this is briefly discussed in section 2 .

The Baryon Asymmetry of the Universe (BAU) is generated via leptogenesis ([28]; for a review, see e.g. [4]) at the $\mathrm{TeV}$ scale. As the temperature of the Universe drops below the mass of the singlet $\psi \mathrm{s}$, their number density is depleted by annihilations and decays. We require this to occur prior to the electroweak phase transition. They can decay to SM neutrinos $\nu$ and a Higgs, or to light singlets $s$ and $\phi$, and CP asymmetries can arise in these decays. Since lepton number is conserved until $\phi$ gets a vev, these lepton asymmetries would be of equal magnitude and opposite sign in the doublets and singlets. The asymmetries can survive in the plasma, once "washout interactions", exchanging lepton number between the doublets and singlets, are out of equilibrium. Section 3.3 estimates that the co-moving number density of $\psi$ s remaining, when this occurs, is "naturally" of order that of WIMPs. So to "naturally" obtain similar relic densities of baryons and WIMPs would require to "naturally" obtain $\mathcal{O}(1) \mathrm{CP}$ asymmetries in the $\psi$ decays. As reviewed in section 3.2, large $\mathrm{CP}$ asymetries can be arranged by taking the $\psi$ masses to be quasi-degenerate [29-31]. The lepton asymmetry in Standard Model neutrinos then can be partially transformed to baryons by non-perturbative SM B $+\mathrm{L}$ violation. Dark matter is assumed to be some other WIMP, with a "usual" relic abundance. So this scenario gives similar abundances of WIMPs and baryon-parents, but similar dark matter and baryon number densities only arise if there is an $\mathcal{O}(1) \mathrm{CP}$ asymmetry in the parent decays. Unfortunately, such a large $\mathrm{CP}$ asymmetry is not easy to obtain in our model. We comment on this scenarios ability to relate the baryon and dark matter densities in section 4 .

\section{Review, notation and masses}

\subsection{Observations}

The mass density of baryons $B$ in the Universe today, as extracted from a canonical $\Lambda C D M$ model [32], is

$$
\frac{m_{p}\left(n_{B}-n_{\bar{B}}\right)}{\rho_{c}}=\Omega_{B} h^{2}=0.02255 \pm 0.0054 \Rightarrow Y_{B} \equiv \frac{\left(n_{B}-n_{\bar{B}}\right)}{s} \simeq 8.7 \times 10^{-11}
$$

where $s=2 \pi^{2} g_{*} T^{3} / 45$ is the entropy density, $\rho_{c}=3 H_{0}^{2} /(8 \pi G)$ is the critical density to obtain a spatially flat Universe, and $H_{0}=74.2 \pm 3.6 \mathrm{~km} / \mathrm{sec} / \mathrm{Mpc}=100 \mathrm{~h} \mathrm{~km} / \mathrm{sec} / \mathrm{Mpc}$. The cold dark matter relic abundance, for the same canonical $\Lambda C D M$ cosmology with three light neutrinos, is

$$
\Omega_{D M} h^{2}=0.1126 \pm 0.0036
$$


so that the ratio of dark matter particles to baryons is

$$
\frac{Y_{D M}}{Y_{B}} \sim 5 \frac{m_{p}}{m_{D M}}
$$

where $m_{p}$ is the proton mass. Since $m_{p}$ mostly arises from QCD, and the dark matter should not have strong interactions, we assume that $m_{p}$ and $m_{D M}$ are unrelated, and focus on obtaining similar baryon and dark matter number densities today: $Y_{B} / 30 \lesssim Y_{D M} \lesssim 30 Y_{B}$. This definition of "similar" allows a dark matter candidate in the mass range accessible to most direct detection experiments.

Notice that the ratio (2.3) could be different in more compliciated cosmological models, such as those with extra sterile neutrinos. The CMB data alone allows the dark matter density to increase with the number of relativistic species present at recombination (counted as the number $N_{\text {eff }}$ of neutrino flavours): $\delta \Omega_{D M} \sim .4\left(\delta N_{\text {eff }} \Omega_{D M} / N_{\text {eff }}\right)$ [32]. Including other observations, such as $H_{0}$, favours $N_{\text {eff }}=4.34_{-0.88}^{+0.86}\left(68 \%\right.$ CL) [32, 33]. An increase in $\Omega_{D M}$ would usefully increase the ratio (2.3), various laboratory neutrino anomalies [25-27] could benefit from light neutrinos other than the three of the Standard Model, and this paper constructs a neutrino mass model with additional light singlets. However, cosmological data also constrains the light neutrino mass scale. An additional eV sterile neutrino that could address the reactor neutrino anomaly should respect cosmological bounds on hot dark matter. It was shown by [33] that such a $\mathrm{eV}$-sterile is cosmologically consistent if there are additional lighter neutrino species to delay matter radiation equality, and an $\mathcal{O}(0.01)$ asymmetry in the $\nu_{e}$ density to allow Big Bang Nucleosynthesis $(\mathrm{BBN})[34,35]$ to produce the observed relic abundances of light elements. Our model allows several sterile species, but the baryon asymmetry is related to the asymmetry in SM neutrinos, so we do not obtain the large $\nu_{e}$ chemical potential required by [33, 35]. Furthermore, if our extra steriles decouple prior to the Electroweak Phase Transition (EPT), their contribution to the radiation density at BBN is suppressed by a factor $g_{*}\left(T_{B B N}\right) / g_{*}\left(T_{E P T}\right)$, where $g_{*}(T)$ is the number of Standard Model degrees of freedom in the plasma at $T$.

\subsection{Models}

An explanation for the ratio (2.3), which has benefited from recent interest [10-19] could be that a single asymmetry controls both the baryon and dark matter relic number densities. If this ratio, given in eq. (2.3), should be exactly one, then a comparatively light cold dark matter candidate is required. A model where the DM gets a mass after the EPT is [36]. The observed difference in mass densitites could also be due to inequivalent cosmological histories after the formation of the asymmetry. Some early discussions of asymmetries in the dark matter density are [7-9, 37]; for a review of recent Asymmetric Dark Matter (ADM) scenarios, see e.g. [38, 39]. Asymmetric leptogenesis models [17-19], and links to Dark Energy and inflation [40,41] also have been explored. ADM models explain why the dark matter and baryon number densities are similar, but not why they have have the observed value.

An alternative approach [20-23], attaches importance to the "WIMP miracle" (see eg $[5,6])$ : the relic abundance of a massive particle, which annihilates with its antiparticle via a weak cross-section, gives $\Omega_{D M} h^{2}$ of order the observed value. To explain 
the ratio (2.3), one then only needs a natural scenario giving a relic number density of baryons minus anti-baryons, which is similar to the relic number density of WIMPS plus anti-WIMPS. It is well-known [1] that to produce an asymmetry, CP violation and nonequilibrium are required, whereas only a departure from thermal equilibrium is required for the particle-antiparticle-symmetric relic density of WIMPs. So if the non-equilibrium is similar in the baryon and dark matter production, and the CP asymmetry for baryogenesis is one, then similar number densities could be expected. For instance, in [20], the baryon asymmetry is produced in the out-of equilibrium annihilations of the dark matter particle, which works for selected mass ranges of the particles involved. In this paper, the lepton asymmetry is produced in decays. The parent particle of the asymmetry first annihilates, then decays out-of-equilibrium, at the $\mathrm{TeV}$-scale. This freezes-in the baryon-parent and dark matter densities at a similar temperature, so the departures from thermal equilibrium in both processes may be similar. Indeed, the co-moving density of a weak-scale unstable particle, when inverse decays freeze out, is similar to the relic density of a weak-scale particle that annihilates. So an $\mathcal{O}(1) \mathrm{CP}$ asymmetry is required to get similar baryon and dark matter densities today.

We construct a TeV-scale model of out-of-equilibrium-decay leptogenesis [28]. Various mechanisms have been studied for leptogenesis ${ }^{1}$ in $\mathrm{TeV}$-scale seesaw models [44], such as degenerate decaying singlets [29-31], or adding extra particles [45-47]. Heavy singlet fermions in the "inverse seesaw" pattern have been studied by various people [48-52]. Our model differs from the usual inverse seesaw in that we have additional light singlets [53], so we can generate a non-zero asymmetry in SM leptons, despite that the model conserves lepton number. Our leptogenesis scenario therefore differs from that of [48], who also study a lepton number conserving scenario, but without extra light singlets. They obtain a sufficient baryon asymmetry by turning off the sphalerons before inverse decays go out of equilibrium, when various asymmetries are present and changing. Here, we explore whether the baryon asymmetry can be obtained in a more "dynamics-independent" way, due to the presence of extra singlets. The Lagrangians of [49] contain "hard" lepton number breaking, so the relevant interactions for leptogenesis are somewhat different, because a net lepton asymmetry in generated. An advantage of the hard breaking is that it can naturally provide the small mass splitting between the heavy decaying singlets [49]. The model of [50] generates the asymmetry in the $\mathrm{TeV}$-scale singlets. We focus on a lepton number conserving model, because it is for such models that a "hidden sector" (as provided by the extra light singlets) is useful for storing an asymmetry [54]. Futhermore, the natural scale of lepton number violation in the inverse seesaw is below the electroweak scale, so if lepton number violation is spontaneous, one could expect it to arise after the electroweak phase transition.

The inverse seesaw [24] has received attention because it generates small neutrino masses due to new particles with TeV-scale masses and couplings of a "natural" size. Such new particles may therefore be kinematically accessible to the LHC [55-57], or induce

\footnotetext{
${ }^{1}$ Low temperature out-of-equilibrium-decay baryogenesis scenarios have also been considered; the analysis of [43] was useful to us.
} 
detectable [58-63] non-unitarity and/or Lepton Flavour Violation. We wish to explore, in models where the small lepton number violation is spontaneous, the prospects of additional light singlets and leptogenesis. A careful study [48] (without additional light singlets $s$ ), showed that the baryon asymmetry can be generated by turning off the sphalerons during the decays. We had hoped that baryogenesis would be easier with additional light singlets, but the parameter space where we estimate that it could work is not large enough to be convincing.

Dark Matter has been studied in radiative inverse seesaw models [64-67], where it can arise more naturally than in the inverse seesaw $[68,69]$. We recall that our model does not aim to provide a WIMP; we suppose that it arises in some other sector of the theory.

\subsection{Notation and masses}

We consider a Lagrangian with several additional singlets, which can spontaneously violate lepton number. The SM doublet leptons $\ell$ have Yukawa couplings to (two or three) "righthanded neutrinos" $N_{R}$, there is some larger number of singlet left-handed leptons $\{S, s\}$, and an $L=-2$ singlet scalar $\phi$. The left-handed singlets who participate in a mass matrix $M$ with the $N_{R}$ are refered to as $\{S\}$, and the remaining ss can acquire masses once $\phi$ develops a vev. Allowing all renormalisable interactions, the Lagrangian, written in twocomponent notation with all the fermions left-handed, is:

$\mathcal{L}=\mathcal{L}_{\mathrm{SM}}+\left\{\lambda^{*}[\ell \tilde{H}] N^{c}+M^{*} N^{c} S+y^{*} \phi S s+\frac{Y^{*}}{2} \phi S S+\frac{X^{*}}{2} \phi s s+\frac{Z^{*}}{2} \phi^{\dagger} N^{c} N^{c}+\right.$ h.c. $\}+V(\phi)$,

$\tilde{H}$ is the Higgs, $[\ell \tilde{H}]=\nu \tilde{H}_{0}-e \tilde{H}_{+}$, and $N^{c}, S$ and $s$ have lepton numbers $L=\{-1,1,1\}$. The potential $V(\phi)$ causes $\phi$ to develop a vev after the electroweak phase transition. We assume that such a potential can be constructed, and consider it no further. We also ignore the resulting majoron [70]. Generation indices are implicit; for simplicity, we suppose two generations of $N, S$ and $s$, so the matrices $\lambda, y, Y, X, Z$ and $M$ are two by two. We denote the eigenvalues of $\lambda$ as $\lambda_{1}, \lambda_{2}$ (and similarly for other matrices). We will see that $\lambda_{1} \sim \lambda_{2}$, $y_{1} \sim y_{2}$ and $M_{1} \sim M_{2}$ to obtain a large enough CP asymmetry for leptogenesis.

Prior to the Electroweak Phase Transition, there are two gauge singlet Dirac fermions, $\psi_{1}, \psi_{2}$ :

$$
\psi_{I}=\left(\begin{array}{c}
S_{I} \\
N_{I}
\end{array}\right),
$$

whose masses $M_{I} \sim \mathrm{TeV}$ are the eigenvalues of $M$. The $M_{I}$ will be taken degenerate to obtain a large enough baryon asymmetry, so when the mass difference is irrelevant, we write $M_{\psi}$.

In the presence of vacuum expectation values $v=\langle H\rangle=174 \mathrm{GeV}$ and $u=\langle\phi\rangle \lesssim v$, the neutral Majorana mass matrix can be written, in two component notation, as

$$
\mathcal{L}_{\text {mass }}=-\frac{1}{2}\left(\nu_{L} N^{c} S s\right)\left[\begin{array}{cccc}
0 & m_{D} & 0 & 0 \\
m_{D}^{T} & \mu_{Z} & M & 0 \\
0 & M^{T} & \mu_{Y} & \mu_{y} \\
0 & 0 & \mu_{y}^{T} & \mu_{X}
\end{array}\right]\left(\begin{array}{c}
\nu_{L} \\
N^{c} \\
S \\
s
\end{array}\right)+\text { h.c. }
$$

where $m_{D}=\lambda v, \mu_{X}=X u, \mu_{y}=y u, \mu_{Z}=Z u$, and $\mu_{Y}=Y u$ are two by two matrices. 
We are interested in the limit where the eigenvalues of $M$ are much larger than the other entries in the mass matrix. In the case of the usual inverse seesaw, which contains no light singlets $s$, the determinant implies that the light active neutrino mass matrix is $\sim m_{D} M^{-1} \mu_{Y} M^{T-1} m_{D}^{T}$. In the case where extra singlets are present, the determinant of the mass matrix in (2.6), in one generation, is $m_{D}^{2}\left(\mu_{Y} \mu_{X}-\mu_{y}^{2}\right)$. We neglect from now on the coupling $Z$, because its contributions to the mass matrix are unimportant, and its effects in the baryogenesis scenario are similar to those of $Y$, which will be unimportant. We will see in section 3.3 that the couplings in $y$ and/or $\lambda$ must be $\operatorname{small}^{2}\left(\lesssim 10^{-5}\right)$, to prevent the washout of the lepton asymmetry. We focus on two simple limiting cases: $y_{i} \ll Y_{i}, X_{i} \lesssim 1$ , which gives Majorana masses for the active neutrinos, and the case $Y_{i}=X_{i}=0$, which gives Dirac masses between the $\nu_{L}$ and $s$.

In the Majorana case with $y_{i} \ll Y_{i}, X_{i}$, the active neutrinos have a usual-inverseseesaw-like mass matrix $m_{\nu} \simeq m_{D} M^{-1} \mu_{Y} M^{T-1} m_{D}^{T}$, whose eigenvalues satisfy

$$
\frac{m_{a t m} m_{\text {sol }}}{\mathrm{eV}^{2}}=0.3\left(\frac{\lambda_{1} \lambda_{2}}{10^{-9}}\right)^{2}\left(\frac{(3 \mathrm{TeV})^{2}}{M_{1} M_{2}}\right)^{2} \frac{Y_{1} Y_{2}\langle\phi\rangle^{2}}{v^{2}}
$$

$\phi$ should have a mass and vev well below the Higgs vev $v=174 \mathrm{GeV}$, to ensure that lepton number is conserved prior to the electroweak phase transition. However, we will be forced to take the vev of order $v$ (to allow $\lambda_{i}$ small enough to give a sufficient abundance of $\psi$ s after freezeout of washout interactions). We will also require $\lambda_{1} \simeq \lambda_{2}$, to obtain a large enough $\mathrm{CP}$ asymmetry, so we will suppose a mild hierarchy in $Y$ to generate the atmospheric solar splitting.

The light steriles $s$ have a mass matrix $\sim \mu_{X}=X\langle\phi\rangle$. In the one generation case, $s$ has a mixing angle with the active neutrino of order $\mu_{y} m_{D} /\left(M \mu_{X}\right)$, which allows the decay $s \rightarrow 3 \nu$ at tree level. We focus on parameters ${ }^{3} X \lesssim Y$, such that the singlets are innocuous: their masses are $\lesssim \mathrm{GeV}$, they annihilate efficiently (to majorons), and otherwise can decay. Since the $s$ s decouple prior to the Electroweak Phase Transition, their temperature at $T \sim \mu_{X}$ is suppressed with respect to the photons, so they should not over-contribute to the radiation density.

The active neutrinos share Dirac masses $\sim m_{D} \mu_{y} / M$ with the singlets $s$, if $X=Y=$ $Z=0$ in the Lagrangian (2.4). We consider this limit only in section 3.5, because it allows more parameter space for our leptogenesis scenario. ${ }^{4}$ By assigning $L=0$ to $\phi$, and -1 to $s$, lepton number clearly is conserved also after both $\phi$ and $H$ get vevs. However, since $\phi$ was introduced to spontaneously break lepton number, it is peculiar to not give it lepton number. We nonetheless assume that $\phi$ does not mix with $H$, and do not discuss potential constraints from Higgs physics.

\footnotetext{
${ }^{2}$ We therefore do not expect bounds on our model from lepton flavour violating processes such as $\mu \rightarrow e \gamma$, or non-unitarity [58-63].

${ }^{3}$ With $X \sim 10^{-10}$, we can obtain a singlet $s$ with $\mathrm{eV}$ mass and $\sim 0.1-0.01$ mixing angle, that could fit the reactor neutrino anomaly. However, we do not explore this parameter space, because out model cannot give the large lepton asymmetry required to make it cosmologically acceptable.

${ }^{4}$ Mixed scenarios can be envisaged, for instance where the atmospheric mass is Dirac, and the solar mass is Majorana. We do not study this tuned example because we estimate that out-of-equilibrium-decayleptogenesis does not occur.
} 


\section{Thermal history}

One of the roles of $\psi$ is to generate the Baryon Asymmetry of the Universe (BAU). In its decays, it can generate an active versus sterile lepton asymmetry and the excess of active leptons will be partially transformed to baryons by sphalerons [42]. This is discussed in section 3.2. The asymmetries produced in $\psi$ decay can survive in the plasma once "washout interactions" (such as inverse decays $\ell H \rightarrow \psi$ and $\bar{\phi} \bar{s} \rightarrow \psi$, and scattering $\ell H \rightarrow \bar{\phi} \bar{s}$ ) go out of equilibrium. This "freeze-out" temperature $T_{\mathrm{BAU}}$ is estimated in section 3.3. The baryon asymmetry present today will be generated in the decays of the $\psi$ s remaining at $T_{\mathrm{BAU}}$.

Decays and inverse decays are not the only interactions which change the $\psi$ number density; $\psi+\bar{\psi}$ can also annihilate, via the coupling $Y$ to $\phi+\bar{\phi}$. The annihilation rate is faster than the decay rate at temperatures just below the $\psi$ mass, so we start by discussing annihilations below in section 3.1. Annihilations and inverse decays will freeze out at similar temperatures.

\section{$3.1 \psi \bar{\psi} \rightarrow \phi \bar{\phi}$ annihilations}

At temperatures $T \gtrsim M_{I}$, an equilibrium abundance of $\psi_{I}$ and $\bar{\psi}_{I}$ will be present, because they can be produced via Yukawa interactions involving $\lambda$ (for instance in $t_{R} \overline{t_{L}} \rightarrow \tilde{H}^{*} \rightarrow \ell \bar{\psi}$, at a rate $\Gamma \sim h_{t}^{2} \lambda^{2} T /(4 \pi)$, which is fast compared to the Universe expansion $H$ ).

As the temperature $T$ drops below their mass, the singlets can annihilate via their $Y$ couplings, or decay to a light lepton and a scalar via their $\lambda$ or $y$ couplings:

$$
\begin{aligned}
\psi_{I}+\bar{\psi}_{J} & \rightarrow \phi+\bar{\phi} \\
\psi_{I} & \rightarrow \ell+\tilde{H}, \bar{s}+\bar{\phi}
\end{aligned}
$$

We focus first on annihilations, because we envisage $1 \sim Y_{i} \gg y_{j}, \lambda_{k} \lesssim 10^{-4}$, which implies that initially, at $T \lesssim M_{I}$, the $\psi_{I}$ s are more likely to annihilate than decay.

The non-relativistic annihilation cross-section in the centre of mass is

$$
\sigma v\left(\psi_{I}+\bar{\psi}_{J} \rightarrow \phi+\bar{\phi}\right)=\frac{\left|\left[Y Y^{\dagger}\right]_{I J}\right|^{2}}{128 \pi M_{\psi}^{2}}
$$

where all the singlet masses are approximated as $M_{\psi}$. We neglect the Sommerfeld enhancement due to $\phi$ exchange ${ }^{5}$ because the $\psi$ s are almost relativistic; the enhancement can be estimated [71] to be a few percent. The number density of $\psi_{I}$ s remaining when annihilations freeze out, and the freeze-out temperature $T_{a n n} \equiv M_{\psi} / z \simeq M_{\psi} / 20$ can be determined from

$$
n_{\psi}\left(T_{a n n}\right) \sigma v \simeq H\left(T_{a n n}\right) \simeq \frac{1.7 \sqrt{g_{*}\left(T_{a n n}\right)} T_{a n n}^{2}}{m_{p l}}
$$

where $g_{*}$ is the number of degrees of freedom in the plasma, and $g_{*}(\mathrm{TeV}) \simeq 100$ in our model.

\footnotetext{
${ }^{5}$ We thank Marco Cirelli for discussions of this issue.
} 

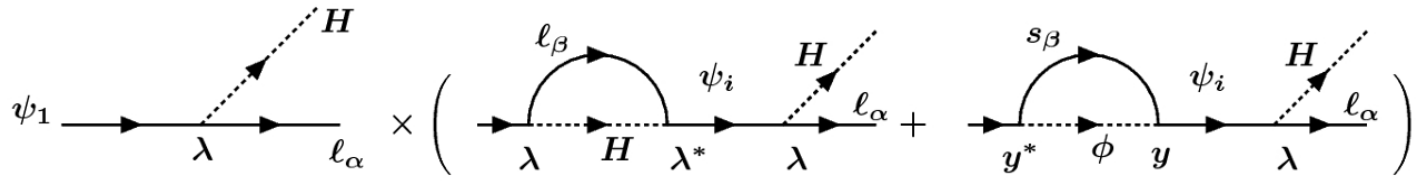

Figure 1. Diagrams contributing to the CP asymmetry with final state $\ell_{\alpha}+H$.

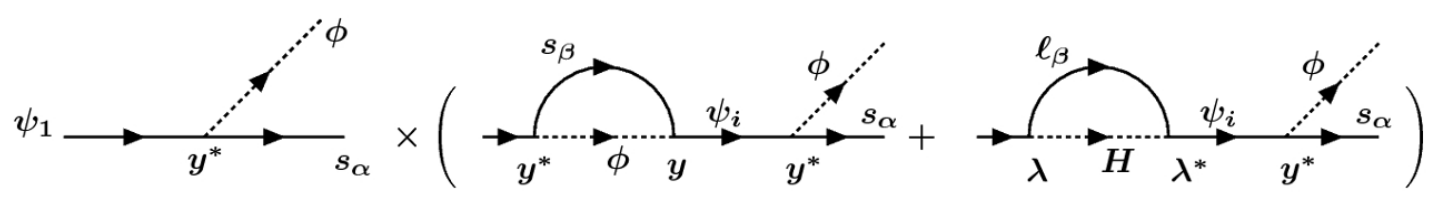

Figure 2. Diagrams contributing to the CP asymmetry with final state $s_{\alpha}+\phi$.

\subsection{Decays and the CP asymmetry}

After the $\psi$ annihilations freeze out, the number density of $\psi$ s and $\bar{\psi}$ will continue to drop, because they decay. The model conserves lepton number, so the total lepton asymmetry produced in these decays is zero. However, since the $\psi$ s decay both to SM particles $H, \ell$, or to singlets $\bar{s}, \bar{\phi}$, equal but opposite lepton asymmetries in the singlet and doublet sectors could be produced. To obtain a baryon to dark matter ratio that is $\mathcal{O}(1)$, these $\mathrm{CP}$ asymmetries will need to be large, so we explore this limit below.

The $\psi_{I}$ s, which carry $L=1$, can decay at tree level to an $\ell_{\alpha}$ and an $H$, or to a $\bar{\phi}$ $(L=2)$ and a $\bar{s}_{\beta}$, at partial rates

$$
\Gamma\left(\psi_{I} \rightarrow \ell_{\alpha}+H\right)=\frac{|\lambda|_{\alpha I}^{2}}{16 \pi} M_{I} \quad, \quad \Gamma\left(\psi_{I} \rightarrow \bar{s}_{\beta}+\bar{\phi}\right)=\frac{|y|_{\beta I}^{2}}{32 \pi} M_{I}
$$

See the first diagrams of figures 1 and 2. These decays are fast, compared to the expansion rate $H$, for $T \lesssim\{|\lambda|,|y|\} \sqrt{M_{I} m_{p l}} / 20$, so at all temperatures relevant to us.

$\mathrm{CP}$ violating asymmetries for a given final state:

$$
\epsilon_{\alpha}^{(I, \ell)}=\frac{\Gamma\left(\psi_{I} \rightarrow \ell_{\alpha} H\right)-\Gamma\left(\bar{\psi}_{I} \rightarrow \bar{\ell}_{\alpha} \bar{H}\right)}{\Gamma\left(\psi_{I} \rightarrow \text { all }\right)+\Gamma\left(\bar{\psi}_{I} \rightarrow a l l\right)} \quad, \quad \epsilon_{\alpha}^{(I, s)}=\frac{\Gamma\left(\psi_{I} \rightarrow \bar{s}_{\alpha} \bar{\phi}\right)-\Gamma\left(\bar{\psi}_{I} \rightarrow s_{\alpha} \phi\right)}{\Gamma\left(\psi_{I} \rightarrow a l l\right)+\Gamma\left(\bar{\psi}_{I} \rightarrow a l l\right)}
$$

can be obtained from the interference of tree with loop diagrams, if the couplings are complex and some particles in the loop can be on-shell. In figures 1 and 2, are drawn the subset of one-loop diagrams which we include in our calculation. We are interested in $\epsilon \rightarrow 1$, which can be obtained in the decays of quasi-degenerate singlets from the illustrated wave-function renormalisation diagrams [29-31], so we neglect the additional vertex corrections diagrams.

We neglect the effects of $X$ and $Y$ couplings in the decay rate and CP asymmetries, despite the hierarchy in magnitudes: $X, Y \sim 1, y, \lambda \sim 10^{-4}$. This is because decays involving $X$ and $Y$ are suppressed by three-body final state phase space, and we were unable to find significant effects of $X$ and $Y$ in the $\mathrm{CP}$ asymmetries.

In the decay of $\psi_{I}$, the total asymmetry in the doublets, summed on lepton flavours $\alpha$, is

$$
\epsilon^{(I, \ell)}=\sum_{\alpha} \epsilon_{\alpha}^{(I, \ell)}=\sum_{\alpha, \beta, J} \frac{\operatorname{Im}\left\{\lambda_{\alpha I} \lambda_{\alpha J}^{*} y_{\beta I} y_{\beta J}^{*}\right\}}{8 \pi\left[2 \lambda^{\dagger} \lambda+y^{\dagger} y\right]_{I I}} \frac{\sqrt{x_{J}}}{1-x_{J}}
$$


where $x_{J}=M_{J}^{2} / M_{I}^{2}$. This contribution arises from the interference of the tree decay with the last loop of figure 1. Similarly, the total asymmetry in the singlets is

$$
\epsilon^{(I, s)}=\sum_{\alpha} \epsilon_{\alpha}^{(I, s)}=\sum_{\alpha, \beta, J} \frac{\operatorname{Im}\left\{y_{\alpha I}^{*} y_{\alpha J} \lambda_{\beta J} \lambda_{\beta I}^{*}\right\}}{8 \pi\left[2 \lambda^{\dagger} \lambda+y^{\dagger} y\right]_{I I}} \frac{\sqrt{x_{J}}}{1-x_{J}}
$$

In both the doublet and singlet sectors, there can be asymmetries in the individual flavours $\alpha$, which arise from the middle diagram of figures 1 and 2. We neglect these contributions, and focus on the flavour-summed singlet and doublet asymmetries of eqns (3.7) and (3.8), because its the total (=flavour-summed) doublet lepton asymmetry which the sphalerons transform to baryons. ${ }^{6}$

The asymmetries given are for the decays of any of the $\psi_{I}$. To obtain a large enough CP asymmetry, at least two of the $\psi_{I}$ must be very degenerate: $M_{I}-M_{J} \sim \Gamma_{I}$, so the $\mathrm{CP}$ asymmetries in the decays of at least two of the $\psi_{I} \mathrm{~s}$ will contribute to the baryon asymmetry. It is therefore fortunate that the $\mathrm{CP}$ asymmetries do not vanish when summed on $I$ : in a two generation model, in the limit where $M_{1} \rightarrow M_{2}$, the CP asymmetries are equal $\epsilon^{(1, \ell)}=\epsilon^{(2, \ell)}$, rather than opposite.

As anticipated, the sum of $\epsilon^{(I, \ell)}$ and $\epsilon^{(I, s)}$ vanishes because the model conserves lepton number. It will therefore be important that no interaction be fast enough to equilibrate the singlet and doublet sectors, as the asymmetries are produced and until the Electroweak Phase Transition. This is discussed in section 3.3.

It is helpful to be able to estimate the size of these CP asymmetries. This scenario of out-of-equilibrium decay leptogenesis at the electroweak scale requires a large $\mathrm{CP}$ asymmetry $\epsilon \gtrsim 0.1$. So from eq. (3.7), all the elements $[\lambda]_{I \alpha}$ and $[y]_{I \beta}$ must be comparable, for all $\alpha, I, \beta$, and the $\psi$ s degenerate: $M_{1}-M_{2} \gtrsim \Gamma$. For phases to maximise the asymmetry, large mixing angles, and comparable eigenvalues $\lambda_{1} \sim \lambda_{2}, y_{1} \sim y_{2}$,

$$
\epsilon^{I} \sim \frac{1}{16 \pi} \frac{y_{1}^{2} \lambda_{1}^{2}}{y_{1}^{2}+2 \lambda_{1}^{2}} \frac{M_{I}}{M_{I}-M_{J}} \leq \frac{1}{4 D}
$$

where the last approximation expresses the mass splitting in units of $\Gamma_{I}$

$$
M_{I}-M_{J}=D \Gamma_{I}
$$

and the coupling constant combination was maximised by taking

$$
y_{1} \sim \sqrt{2} \lambda_{1}
$$

Recall that the formulae (3.7) and (3.8) are valid for $M_{I}-M_{J}>\Gamma_{I}$, so $\epsilon \sim 0.1$ is barely consistent. Since the mass $M$ is the only interaction linking the singlet and doublet sector, loop corrections should not destabilise the small splitting.

\footnotetext{
${ }^{6}$ The flavour asymmetries can nonetheless generate the observed baryon asymmetry, due to timedependent effects [48]
} 


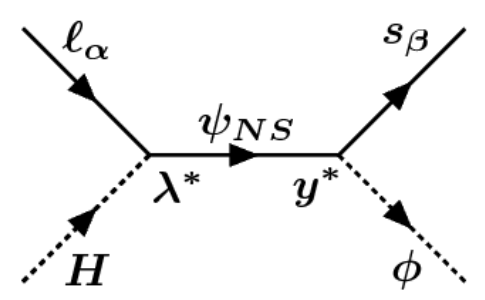

Figure 3. Dangerous scattering process $\ell+H \rightarrow \bar{s}+\bar{\phi}$

\subsection{Washout of the asymmetry by inverse decays and scattering}

As the $\psi$ s decay, they produce an asymmetry in doublet leptons. This asymmetry will survive, and be redistributed throughout the plasma by the interactions in chemical equilbrium $[72,73]$, if all the interactions which can destroy this asymmetry are out of equilbrium. Such destructive interactions are refered to as "washout interactions", and in our model, are the inverse decays $\ell H \rightarrow \psi$ and $\bar{\phi} \bar{s} \rightarrow \psi$, which transfer lepton number back to the $\psi$ s, and the $H \ell \rightarrow \bar{\phi} \bar{s}$ scattering (see figure 3) which exchanges lepton number between the doublets and light singlets. We follow the tradition ${ }^{7}$ of treating the on-shell- $\psi$ part part of figure 3 as inverse decays, and including the remaining "Real Intermediate State -subtracted" part as scattering far below the $\psi$ pole.

For making estimates, it is convenient to have thermally averaged rates, as opposed to the reaction densities ${ }^{8}$ used in Boltzmann Equations. So we define the thermally averaged inverse decay rates as

$$
\left\langle\Gamma\left(\ell_{\alpha} H \rightarrow \psi_{I}\right)\right\rangle=\frac{\gamma\left(\Psi_{I} \rightarrow \ell_{\alpha} H\right)}{n_{\ell}(T)} \simeq \frac{n_{\psi}^{(e q)}(T)}{n_{\ell}(T)} \Gamma\left(\Psi_{I} \rightarrow \ell_{\alpha} H\right)
$$

where $n_{\ell}(T) \simeq g_{\ell} T^{3} / \pi^{2}$, the $\psi$ decay rates are given in eq. (3.5), and the equilibrium abundance of a non-relativistic particle $X$ is

$$
n_{X}^{e q}(T) \simeq g_{X}\left[\frac{m_{X} T}{2 \pi}\right]^{3 / 2} e^{-m_{X} / T}
$$

where $g_{X}$ is the number of spin degrees of freedom of $X$ (notice that our number densities describe particles, not the particles + anti-particles). Comparing the inverse decay rate of eq. (3.12) and the annihilation rate of (3.4) to the Hubble expansion, shows that they freeze out at a similar temperature

$$
T_{\mathrm{BAU}} \simeq \frac{M}{20}
$$

The annihilations, discussed in section 3.1, will freeze out first for

$$
\left|\left[Y Y^{\dagger}\right]_{I I}\right|^{2}<\frac{M_{\psi}^{3}}{T^{3}} 2 \pi^{2}\left|y_{\beta I}\right|^{2}, 4 \pi^{2}\left|\lambda_{\alpha I}\right|^{2}
$$

\footnotetext{
${ }^{7}$ See [4] for a discussion, and references, of this curious separation.

${ }^{8}$ See, for instance, [4] for definitions.
} 
We will see that this condition is satisfied, so annihilations stop depleting the $\psi$ number density before the inverse decays allow a lepton asymmetry to survive.

The number density of $\psi \mathrm{s}$ remaining when both inverse decays $\left\langle\Gamma\left(\ell H \rightarrow \psi_{I}\right)\right\rangle$ and $\left\langle\Gamma\left(\phi s \rightarrow \overline{\psi_{I}}\right)\right\rangle$ are out of equilibrium is

$$
n_{\psi}\left(T_{\mathrm{BAU}}\right)=\min \left\{\frac{H\left(T_{\mathrm{BAU}}\right)}{\Gamma\left(\Psi_{I} \rightarrow \ell H\right)} n_{\ell}\left(T_{\mathrm{BAU}}\right), \frac{H\left(T_{\mathrm{BAU}}\right)}{\Gamma\left(\Psi_{I} \rightarrow \bar{\phi} \bar{s}\right)} n_{s}\left(T_{\mathrm{BAU}}\right)\right\}
$$

where $\Gamma$ and $H$ are given in equations (3.5) and (3.4).

To protect a doublet lepton asymmetry, the inverse decays from the singlet and doublet sectors must both be out of equilibrium. To see this, recall that the heavy Dirac $\psi$ s have $L=1$, and there are equal numbers of $\psi \mathrm{s}$ and $\bar{\psi} \mathrm{s}$ to begin. As they decay, opposite lepton asymmetries develop in the doublet leptons and light singlet sector. Consider now the case that inverse decays from the doublets are out of equilibrium, but that the asymmetry in light singlets can be transfered back to the heavy Dirac leptons by $\phi s \rightarrow \overline{\psi_{I}}$. Then the anti-asymmetry from the light singlet sector is transfered to the $\psi$ s and $\bar{\psi}$ s, who transmit it to the doublets via decays, which are always in equilibrium.

Away from the $\psi$ mass pole, the scattering cross-section for $\ell+H \rightarrow \bar{s}+\bar{\phi}$ is

$$
\sigma\left(\ell_{\alpha}+H \rightarrow \bar{s}_{\beta}+\bar{\phi}\right)=\frac{\left|y_{\beta I} \lambda_{\alpha I}\right|^{2}}{32 \pi} \frac{M_{I}^{2}}{\left(s-M_{I}^{2}\right)^{2}} \rightarrow \frac{\left|y_{\beta I} \lambda_{\alpha I}\right|^{2}}{32 \pi M_{I}^{2}}
$$

where, after the arrow, the internal line momentum is neglected. This is an acceptable approximation at temperatures $T \lesssim M_{I} / 20$, where the inverse decays are out of equilibrium. With this approximation, the thermally averaged scattering rate $\Gamma \sim \sigma n_{\ell}$ is out of equilibrium for

$$
\left|y_{\beta I} \lambda_{\alpha I}\right|^{2} \lesssim 4 \times 10^{-11} \frac{M_{I}}{3 \mathrm{TeV}} .
$$

\subsection{Putting it all together in the Majorana case}

The "washout" interactions go out of equilibrium at $T_{\mathrm{BAU}}$ (given in eq. (3.14)), after which an asymmetry in the SM leptons is produced. This should occur prior to the electroweak phase transition, which we take to occur at $T_{E P T} \sim 150 \mathrm{GeV}[74,75]$. Requiring $T_{\mathrm{BAU}}>$ $T_{E P T}$, imposes a lower bound on the singlet mass scale

$$
M \gtrsim 3 \mathrm{TeV}
$$

There is also an upper bound on $M$, as a function of $\lambda$ and $Y$, from requiring that the inverse seesaw give the observed neutrino mass differences. This condition was given in eq. (2.7). Since a large enough CP asymmetry requires large mixing angles and comparable eigenvalues in $\lambda$ and $y$, we can restrict to the one generation case:

$$
m_{\text {atm }}=0.59\left(\frac{\lambda_{2}}{10^{-9}}\right)^{2}\left(\frac{(3 \mathrm{TeV})}{M_{2}}\right)^{2} \frac{Y_{2}\langle\phi\rangle}{v} \mathrm{eV}
$$

And finally, the requirement that $\psi \bar{\psi} \rightarrow \phi \bar{\phi}$ annihilations freeze-out before the inverse decays $\ell_{\alpha} H \rightarrow \psi$, imposes the relation between $Y$ and $\lambda$ given in eq. (3.15). Expressed in 


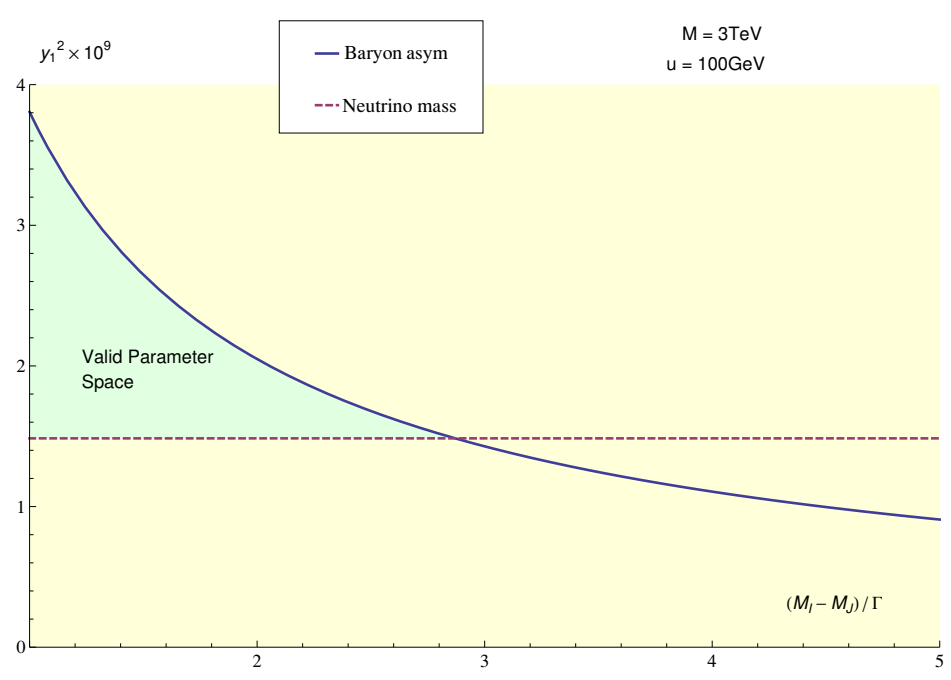

Figure 4. Available parameter space generating the observed baryon asymmetry and neutrino masses, of Majorana type, in the inverse seesaw model with extra light singlets, described by the Lagrangian (2.4).

terms of eigenvalues, we obtain

$$
Y_{2}^{4} \lesssim 10^{-3}\left(\frac{\lambda_{2}^{2}}{10^{-9}}\right)
$$

Electroweak sphalerons will partially transform the $B-L$ asymmetry produced in SM fermions, $Y_{B-L}$, to a baryon asymmetry $[72,73] Y_{B}=(12 / 37) Y_{B-L}$. So the baryon asymmetry produced in our model will be of order

$$
Y_{B} \simeq \frac{12}{37} \frac{n_{\ell}-n_{\bar{\ell}}}{s} \simeq \frac{12}{37} \frac{4 n_{\psi}\left(T_{\mathrm{BAU}}\right)}{s} \epsilon \simeq 4 \times 10^{-10} \frac{M_{\psi}}{3 \mathrm{TeV}} \frac{10^{-9}}{\left|\lambda_{2}\right|^{2}} \frac{1}{D}
$$

where $4 n_{\psi}=\Sigma_{I}\left(n_{\psi_{I}}+n_{\bar{\psi}_{I}}\right)$, and we used eqns (3.16) and (3.9). It is marginally possible to obtain the atmospheric mass difference and the observed baryon asymmetry, with $M=$ $3 \mathrm{TeV}, Y_{2}$ saturating eq. (3.21), and $\langle\phi\rangle \lesssim v$. In figure 4, the available parameter space is plotted as a function $\left|y_{1}\right|^{2} / 10^{-9}$ in eq. (3.10).

\subsection{Dirac limit}

As seen in the section above, for Majorana light neutrino masses, the parameter space of our model is very restricted. In this section, we explore the Dirac limit of the light neutrino mass matrix (2.6), where all Majorana mass terms are zero, $\mu_{A} \rightarrow 0, A \in\{X, Y, Z\}$. Without Majorana mass terms one can redefine the lepton number of $\left\{N^{c}, S, s, \phi\right\}$ to be $\{-1,1,-1,0\}$ so that also after the phase transition of $\phi$ lepton number is conserved. Neutrinos become Dirac particles with $s_{I}$ being their right handed Dirac partners. The light mass eigenvalue is

$$
m_{\nu}^{D}=\frac{m_{D} \mu_{y}}{M}
$$


which is only suppressed by $\frac{1}{M}$ compared to $\frac{1}{M^{2}}$ in the Majorana case. This will significantly enhance our allowed parameter space. But it is also clear that hirarchy in neutrino masses must now come from a hierarchy in the Yukawa eigenvalues $\lambda_{1}<\lambda_{2}$, and/or the same for $y$.

Before looking at the parameter space in the Dirac model, we shall look at its thermal history, comparing to the Majorana case. The first thing to realize is, that annihilation processes do not exist. The only processes that washout $C P$ asymmetry are scattering, as in figure (3) and inverse decays which, like in the Majorana case, determine the moment of efficient $C P$ violation. We assume the inverse decay rates from the various flavours are comparable, despite the mild hierarchy in $\lambda$ and/or $y$. After the inverse decays become slow, all heavy parent particles will decay and produce a $C P$ asymmetry that is not washed-out, so that the first part of equation (3.22) remains valid. Therefore the thermal history in the Dirac limit does not differ significantly from the Majorana case. The value of $\epsilon$, however, will be reduced, because of the Yukawa hierarchy. To be concrete, in figure 5, we take $\epsilon=\frac{1}{16 D}$ (compare to the Majorana case, eq. (3.9)).

One should also have a careful look at Big Bang Nucleosynthesis because it restricts the number of thermalised neutrinos at $T \sim M e V: N_{\nu} \lesssim 4$. It was shown in [76] that right handed Dirac neutrinos are not in equilibrium at $\mathrm{T} \sim \mathrm{MeV}$ because their interaction rates are suppressed by a factor $\left(\frac{m_{\nu}}{T}\right)^{2}$ compared to that of left handed neutrinos. Consequently the number of neutrinos in thermal equilibrium at BBN is not changed by our model with respect to the Standard Model.

Let us now turn to the parameter space of the Dirac limit. In the Majorana case the allowed parameter space is very small because asymmetry production favours small $y$, in order to produce a big density of $\psi$ when inverse decays freeze out which can then be transformed into enough baryon asymmetry, even if $\epsilon<1$. But neutrino masses prefer big $y$ couplings, because the neutrino mass term is already suppressed by $1 / M^{2}$. This forces us to take quite large values for $u$, the vev of $\phi$ in order to enhance neutrino masses without limiting asymmetry production. In the Dirac limit neutrino masses are less suppressed, so that the conflict between baryon asymmetry and neutrino masses is less critical. We can realize our model with moderate values for $u \approx 6 \mathrm{GeV}$, far away from electroweak phase transition, while parent masses are at $M=3 \mathrm{TeV}$ and do not have to be more degenerate than $M_{I}-M_{J} \approx 5 \Gamma\left(\psi_{I} \rightarrow\right.$ all $)$. These values were obtained for a ideal constellation of our parameters, i.e. all phases in our Yukawa couplings $\lambda$ and $y$ are of order one and that

$2 \lambda_{2}^{2} \sim y_{2}^{2}$. However in this Dirac limit small variations from these ideal conditions are possible as the parameter space is big enough to compensate for non ideal conditions. A plot of possible parameter space is given in figure (5).

\section{Discussion}

This paper considered baryogenesis at the electroweak scale, in an out-of-equilibrium decay scenario. This was implemented in an inverse seesaw model with extra light singlets $s$, described by the Lagrangian (2.4). The aim was to relate the baryon asymmetry to the dark matter abundance, and possibly fit various neutrino anomalies with the extra singlets. Similar Dark Matter and baryon abundances were supposed to arise, because, if the Dark 


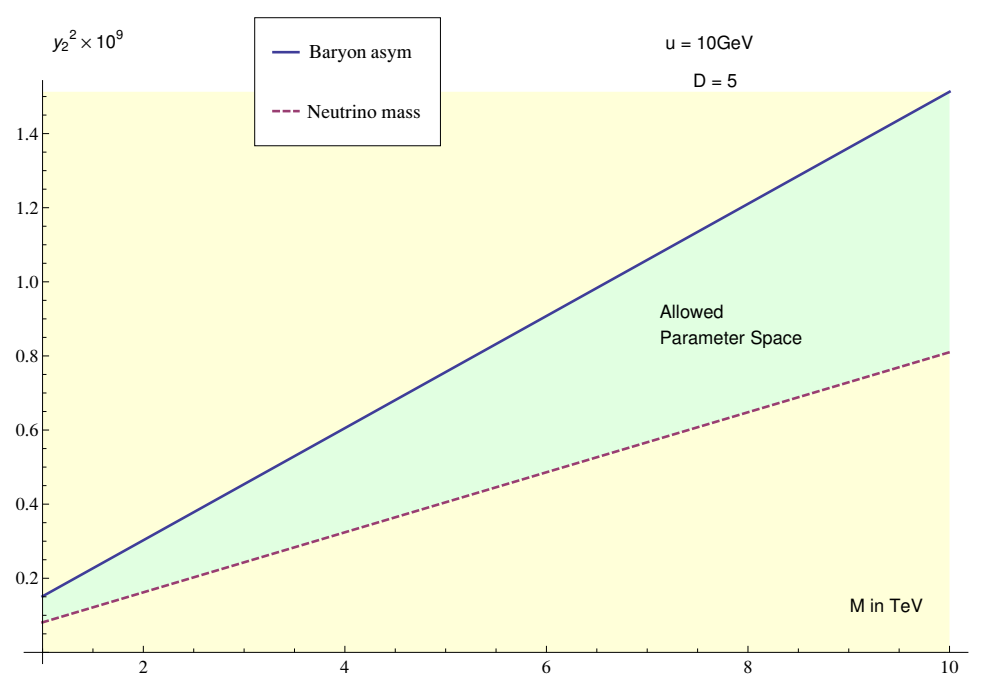

Figure 5. Constraints from the Baryon asymmetry and neutrino masses on $y_{2}^{2}$ as a function of the parent particle mass scale $M_{\psi}$ give the allowed parameter space in the Dirac limit. The upper bound from Baryon asymmetry (solid line) varies with the degeneracy $M_{I}-M_{J} \approx D \Gamma\left(\psi_{I} \rightarrow\right.$ all $)$. For less degenerate parent particles, the slope reduces and the allowed parameter space shrinks. The dashed line is the lower bound from neutrino masses and varies with the vacuum expectation value $u$. For smaller $u$, the slope rises and the parameter space shrinks.

Matter is made of WIMPs, and the baryon asymmetry produced in electroweak-scale out-ofequilibrium decay, then both relic densities are controlled by electroweak-scale interactions going out of equilibrium. The baryon asymmetry is also proportional to a CP asymmetry $\epsilon$, see eq. (3.22).

The scenario for generating the baryon asymmetry in our model is outlined at the end of the introduction. It does not work very well. The first difficulty is that it does not naturally give the large $\mathrm{CP}$ asymmetry that is required, contrary to models with explicit lepton number violation (reviewed in section 2.2). More importantly, the allowed parameter space where it produces a sufficient baryon asymmetry and correct neutrino masses is tiny (see figure 4), and unattractive. It requires a small mass splitting between the heavy singlets $M_{2}-M_{1} \simeq \Gamma$, but the formula for $\epsilon$, eq. (3.7), becomes unreliable in this limit. Also, the inverse seesaw is attractive because it allows new LHC-scale singlet fermions with large Yukawa couplings, and spontaneous lepton number violation due to a small vev. However, our model requires small yukawas $\lambda$ and $y$, to ensure that interactions which wash out the baryon asymmetry freeze out soon enough. This forces a large lepton number violating vev, of order the Higgs vev. The masses of the extra light singlets $s$ are arbitrary, but would be $\lesssim \mathrm{GeV}$, unless a steep hierarchy is tuned into the couplings (see discussion after eq. (2.7)). A larger successful parameter space is obtained (see figure 5), if the Majorana couplings of the inverse seesaw model are set to zero. Then the active neutrinos obtain Dirac masses with the light steriles. However, then the motivation for the singlet scalar (whose vev spontaneouly broke lepton number in the Majorana case), is no longer clear. Since it no longer carries lepton number, it could mix with the Standard Model Higgs, and some analysis would be required to determine its experimental signatures. 
Despite the flaws in our model, it could be unsurprising, if not natural, to obtain similar baryon and dark matter relic densities, when the dark matter are WIMPs, and the baryon asymmetry is produced in out-of-equilibrium decay. In such scenarios, the baryon asymmetry is produced after the freeze-out of washout interactions. This condition can be roughly estimated as

$$
\Gamma(\text { all } \rightarrow \psi) \simeq \Gamma(\psi \rightarrow \text { all }) e^{-M / T} \lesssim H\left(T_{\mathrm{BAU}}\right)
$$

where $\psi$ is the decaying parent of the $\mathrm{BAU}$, of mass $M$, and $H$ is the Hubble expansion rate. Notice that inverse decays are Boltzmann-suppressed, because the light decay products have difficulty to find, in the thermal bath, the energy to produce a heavy $\psi$.

This Boltzmann suppression is reminiscent of the freezeout of annihilations of a WIMP $\chi$, which can be estimated to occur when

$$
n_{\chi}\left(T_{D M}\right)\langle\sigma v\rangle \lesssim H\left(T_{D M}\right)
$$

The $e^{-m / T}$ appears here in equilibrium number density of a non-relativistic particle, see eq. (3.13).

From these two estimates, it is straightforward to estimate the ratio of co-moving densities $^{9}$ of BAU parents $\psi$ to WIMPs $\chi$ as

$$
\frac{Y_{\psi}}{Y_{\chi}} \simeq \frac{1}{(2 \pi z)^{3 / 2}} \frac{g^{4}}{\lambda^{2}} \frac{M_{\psi}}{m_{\chi}} \sqrt{\frac{g\left(T_{D M}\right)}{g\left(T_{\mathrm{BAU}}\right)}}
$$

where the decay rates and annihiation cross-section were normalised to the relevant particle masses $\Gamma \equiv \lambda^{2} M_{\psi} /(8 \pi)$, and $\langle\sigma v\rangle \equiv g^{4} /\left(8 \pi m_{\chi}^{2}\right)$, and we assume that the baryon-parents and WIMPs freeze out at comparable values of $z=m / T \simeq 25$. At a given mass scale, this shows that the co-moving number density of BAU-parents can be easily of the same order as that of particles which annihilate. So weak-scale BAU parents naturally have the same abundance as weak-scale dark matter.

However, all BAU parents do not neccessarily produce baryons. On average, a parent produces $\epsilon$ baryons, where $\epsilon$ parametrises CP violation, and is suppressed by a loop factor, mixing angles and possibly small couplings. This could suggest that $\epsilon$ is naturally $\lesssim 10^{-3}$. Eq. (4.3) predicts

$$
\frac{n_{B}}{n_{D M}} \sim \epsilon \frac{Y_{\psi}}{Y_{\chi}}=C \epsilon \frac{M_{\psi}}{m_{\chi}}
$$

where the coefficient $C$ could be $\mathcal{O}(1)$, for $\lambda^{2} \ll g^{4}$. So to obtain $n_{B} \sim n_{D M}$ requires $\epsilon \sim 1$ for $m_{\chi} \sim M_{\psi}$, or $m_{\chi}<M_{\psi}$ for $\epsilon<1$. It could be reasonable to suppose that the WIMP is an electroweak-scale particle, with gauge couplings $g$, and that the baryonparent is a flavour-scale particle, with flavoured couplings $\lambda$, in which case, such a ratio of couplings could be credible. Then for "natural" values of the CP asymmetry $\epsilon \lesssim 10^{-3}$, and the flavour-scale $M_{\psi} \gtrsim 100 \mathrm{TeV}$, the baryon and WIMP number densities today are "naturally" comparable.

\footnotetext{
${ }^{9}$ Note that the WIMPs and BAU-parents may never simultaneously have this ratio of densities; the physically relevant ratio is $n_{b} / n_{\chi}$.
} 


\section{Acknowledgments}

We thank Steve Abel for participating in the genesis of this project, Nuria Rius for discussions and important comments, Christopher Smith for many useful conversations, and Marco Cirelli for a relevant question. S.D. and M.E. acknowledge partial support from the European Union FP7 ITN INVISIBLES (Marie Curie Actions, PITN- GA-2011- 289442).

Open Access. This article is distributed under the terms of the Creative Commons Attribution License which permits any use, distribution and reproduction in any medium, provided the original author(s) and source are credited.

\section{References}

[1] A.D. Sakharov, Violation of CP invariance, $C$ asymmetry, and barion asymmetry of the universe, Pisma Zh. Eksp. Teor. Fiz. 5 (1967) 32.

[2] A.D. Dolgov, NonGUT baryogenesis, Phys. Rept. 222 (1992) 309 [INSPIRE].

[3] V. Rubakov and M. Shaposhnikov, Electroweak baryon number nonconservation in the early universe and in high-energy collisions, Usp. Fiz. Nauk 166 (1996) 493 [Phys. Usp. 39 (1996) 461] [hep-ph/9603208] [INSPIRE].

[4] S. Davidson, E. Nardi and Y. Nir, Leptogenesis, Phys. Rept. 466 (2008) 105 [arXiv: 0802.2962] [INSPIRE].

[5] G. Jungman, M. Kamionkowski and K. Griest, Supersymmetric dark matter, Phys. Rept. 267 (1996) 195 [hep-ph/9506380] [INSPIRE].

[6] G. Bertone, D. Hooper and J. Silk, Particle dark matter: evidence, candidates and constraints, Phys. Rept. 405 (2005) 279 [hep-ph/0404175] [INSPIRE].

[7] P. Hut and K.A. Olive, A cosmological upper limit on the mass of heavy neutrinos, Phys. Lett. B 87 (1979) 144 [INSPIRE].

[8] K. Griest and D. Seckel, Cosmic asymmetry, neutrinos and the Sun, Nucl. Phys. B 283 (1987) 681 [Erratum ibid. B 296 (1988) 1034] [InSPIRE].

[9] S. Nussinov, Technocosmology: could a technibaryon excess provide a 'natural' missing mass candidate?, Phys. Lett. B 165 (1985) 55 [INSPIRE].

[10] D. Hooper, J. March-Russell and S.M. West, Asymmetric sneutrino dark matter and the $\Omega_{b} / \Omega_{D M}$ puzzle, Phys. Lett. B 605 (2005) 228 [hep-ph/0410114] [InSPIRE].

[11] M.L. Graesser, I.M. Shoemaker and L. Vecchi, Asymmetric WIMP dark matter, JHEP 10 (2011) 110 [arXiv:1103.2771] [INSPIRE].

[12] S.B. Gudnason, C. Kouvaris and F. Sannino, Dark matter from new technicolor theories, Phys. Rev. D 74 (2006) 095008 [hep-ph/0608055] [INSPIRE].

[13] D.E. Kaplan, M.A. Luty and K.M. Zurek, Asymmetric dark matter, Phys. Rev. D 79 (2009) 115016 [arXiv:0901.4117] [INSPIRE].

[14] A. Belyaev, M.T. Frandsen, S. Sarkar and F. Sannino, Mixed dark matter from technicolor, Phys. Rev. D 83 (2011) 015007 [arXiv: 1007.4839] [InSPIRE].

[15] J. March-Russell and M. McCullough, Asymmetric dark matter via spontaneous co-genesis, JCAP 03 (2012) 019 [arXiv:1106.4319] [INSPIRE]. 
[16] K. Kamada and M. Yamaguchi, Asymmetric dark matter from spontaneous cogenesis in the supersymmetric standard model, Phys. Rev. D 85 (2012) 103530 [arXiv:1201.2636] [INSPIRE].

[17] E.J. Chun, Minimal dark matter and leptogenesis, JHEP 03 (2011) 098 [arXiv:1102.3455] [INSPIRE].

[18] Z. Kang, J. Li, T. Li, T. Liu and J. Yang, Asymmetric sneutrino dark matter in the NMSSM with minimal inverse seesaw, arXiv:1102.5644 [INSPIRE].

[19] A. Falkowski, J.T. Ruderman and T. Volansky, Asymmetric dark matter from leptogenesis, JHEP 05 (2011) 106 [arXiv:1101.4936] [INSPIRE].

[20] Y. Cui, L. Randall and B. Shuve, A WIMPy baryogenesis miracle, JHEP 04 (2012) 075 [arXiv: 1112.2704] [INSPIRE].

[21] J. McDonald, Simultaneous generation of WIMP miracle-like densities of baryons and dark matter, Phys. Rev. D 84 (2011) 103514 [arXiv:1108.4653] [INSPIRE].

[22] J. McDonald, Baryomorphosis: relating the baryon asymmetry to the 'WIMP miracle', Phys. Rev. D 83 (2011) 083509 [arXiv: 1009.3227] [InSPIRE].

[23] J. McDonald, Right-handed sneutrino condensate cold dark matter and the baryon-to-dark matter ratio, JCAP 01 (2007) 001 [hep-ph/0609126] [INSPIRE].

[24] M. Gonzalez-Garcia and J. Valle, Fast decaying neutrinos and observable flavor violation in a new class of majoron models, Phys. Lett. B 216 (1989) 360 [INSPIRE].

[25] T. Mueller et al., Improved predictions of reactor antineutrino spectra, Phys. Rev. C 83 (2011) 054615 [arXiv:1101.2663] [INSPIRE].

[26] G. Mention et al., The reactor antineutrino anomaly, Phys. Rev. D 83 (2011) 073006 [arXiv:1101.2755] [INSPIRE].

[27] P. Huber, On the determination of anti-neutrino spectra from nuclear reactors, Phys. Rev. C 84 (2011) 024617 [Erratum ibid. C 85 (2012) 029901] [arXiv:1106.0687] [INSPIRE].

[28] M. Fukugita and T. Yanagida, Baryogenesis without grand unification, Phys. Lett. B 174 (1986) 45 [INSPIRE].

[29] L. Covi and E. Roulet, Baryogenesis from mixed particle decays, Phys. Lett. B 399 (1997) 113 [hep-ph/9611425] [INSPIRE].

[30] A. Pilaftsis, Heavy Majorana neutrinos and baryogenesis, Int. J. Mod. Phys. A 14 (1999) 1811 [hep-ph/9812256] [inSPIRE].

[31] A. Pilaftsis and T.E. Underwood, Resonant leptogenesis, Nucl. Phys. B 692 (2004) 303 [hep-ph/0309342] [INSPIRE].

[32] WMAP collaboration, E. Komatsu et al., Seven-year Wilkinson Microwave Anisotropy Probe (WMAP) observations: cosmological interpretation, Astrophys. J. Suppl. 192 (2011) 18 [arXiv:1001.4538] [INSPIRE].

[33] J. Hamann, S. Hannestad, G.G. Raffelt and Y.Y. Wong, Sterile neutrinos with eV masses in cosmology: how disfavoured exactly?, JCAP 09 (2011) 034 [arXiv:1108.4136] [INSPIRE].

[34] F. Iocco, G. Mangano, G. Miele, O. Pisanti and P.D. Serpico, Primordial nucleosynthesis: from precision cosmology to fundamental physics, Phys. Rept. 472 (2009) 1 [arXiv: 0809.0631] [INSPIRE]. 
[35] A. Mirizzi, N. Saviano, G. Miele and P.D. Serpico, Light sterile neutrino production in the early universe with dynamical neutrino asymmetries, Phys. Rev. D 86 (2012) 053009 [arXiv: 1206.1046] [INSPIRE].

[36] N. Okada and O. Seto, Originally asymmetric dark matter, Phys. Rev. D 86 (2012) 063525 [arXiv: 1205.2844] [INSPIRE].

[37] G.R. Farrar and G. Zaharijas, Dark matter and the baryon asymmetry, Phys. Rev. Lett. 96 (2006) 041302 [hep-ph/0510079] [INSPIRE].

[38] M.T. Frandsen and S. Sarkar, Light asymmetric dark matter, DESY-PROC-2010-03 (2010).

[39] M.T. Frandsen, Asymmetric DM and DM connection to baryogenesis, talk given at the Dark matter underground and in the heavens (DMUH11), July 188-29, CERN, Switzerland (2011).

[40] H. Guo and P. Hung, Shadow fermions, messenger scalars and leptogenesis, Nucl. Phys. B $\mathbf{8 1 4}$ (2009) 76 [arXiv:0810.3341] [INSPIRE].

[41] C. Arina, J.-O. Gong and N. Sahu, Unifying darko-lepto-genesis with scalar triplet inflation, Nucl. Phys. B 865 (2012) 430 [arXiv: 1206.0009] [InSPIRE].

[42] V. Kuzmin, V. Rubakov and M. Shaposhnikov, On the anomalous electroweak baryon number nonconservation in the early universe, Phys. Lett. B 155 (1985) 36 [INSPIRE].

[43] M. Claudson, L.J. Hall and I. Hinchliffe, Cosmological baryon generation at low temperatures, Nucl. Phys. B 241 (1984) 309 [INSPIRE].

[44] T. Hambye, Leptogenesis at the TeV scale, Nucl. Phys. B 633 (2002) 171 [hep-ph/0111089] [INSPIRE].

[45] S.K. Kang and C. Kim, Extended double seesaw model for neutrino mass spectrum and low scale leptogenesis, Phys. Lett. B 646 (2007) 248 [hep-ph/0607072] [INSPIRE].

[46] M. Hirsch, J. Valle, M. Malinsky, J. Romao and U. Sarkar, Thermal leptogenesis in extended supersymmetric seesaw, Phys. Rev. D 75 (2007) 011701 [hep-ph/0608006] [INSPIRE].

[47] E.J. Chun, TeV leptogenesis in Z-prime models and its collider probe, Phys. Rev. D 72 (2005) 095010 [hep-ph/0508050] [INSPIRE].

[48] M. Gonzalez-Garcia, J. Racker and N. Rius, Leptogenesis without violation of B-L, JHEP 11 (2009) 079 [arXiv:0909.3518] [INSPIRE].

[49] S. Blanchet, T. Hambye and F.-X. Josse-Michaux, Reconciling leptogenesis with observable $\mu \rightarrow$ e r rates, JHEP 04 (2010) 023 [arXiv:0912.3153] [INSPIRE].

[50] F.-X. Josse-Michaux and E. Molinaro, A common framework for dark matter, leptogenesis and neutrino masses, Phys. Rev. D 84 (2011) 125021 [arXiv:1108.0482] [INSPIRE].

[51] J. Garayoa, M. Gonzalez-Garcia and N. Rius, Soft leptogenesis in the inverse seesaw model, JHEP 02 (2007) 021 [hep-ph/0611311] [INSPIRE].

[52] S. Blanchet, P.B. Dev and R. Mohapatra, Leptogenesis with TeV scale inverse seesaw in SO(10), Phys. Rev. D 82 (2010) 115025 [arXiv: 1010.1471] [INSPIRE].

[53] H. Zhang, Light sterile neutrino in the minimal extended seesaw, Phys. Lett. B 714 (2012) 262 [arXiv:1110.6838] [InSPIRE].

[54] S. Dodelson and L.M. Widrow, Baryogenesis in a baryon symmetric universe, Phys. Rev. D 42 (1990) 326 [inSPIRE]. 
[55] F. del Aguila and J. Aguilar-Saavedra, Distinguishing seesaw models at LHC with multi-lepton signals, Nucl. Phys. B 813 (2009) 22 [arXiv:0808.2468] [INSPIRE].

[56] A. Akeroyd, C.-W. Chiang and N. Gaur, Leptonic signatures of doubly charged Higgs boson production at the LHC, JHEP 11 (2010) 005 [arXiv: 1009.2780] [INSPIRE].

[57] A. Das and N. Okada, Inverse seesaw neutrino signatures at LHC and ILC, arXiv:1207.3734 [INSPIRE].

[58] M. Malinsky, T. Ohlsson, Z.-z. Xing and H. Zhang, Non-unitary neutrino mixing and CP-violation in the minimal inverse seesaw model, Phys. Lett. B 679 (2009) 242 [arXiv: 0905.2889] [INSPIRE].

[59] F. Deppisch, T. Kosmas and J. Valle, Enhanced $\mu^{-}-e^{-}$conversion in nuclei in the inverse seesaw model, Nucl. Phys. B 752 (2006) 80 [hep-ph/0512360] [INSPIRE].

[60] W. Abdallah, A. Awad, S. Khalil and H. Okada, Muon anomalous magnetic moment and

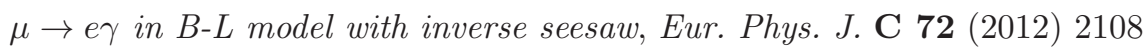
[arXiv: 1105.1047] [INSPIRE].

[61] P.B. Dev and R. Mohapatra, TeV scale inverse seesaw in $\mathrm{SO}(10)$ and leptonic non-unitarity effects, Phys. Rev. D 81 (2010) 013001 [arXiv:0910.3924] [InSPIRE].

[62] M. Hirsch, T. Kernreiter, J. Romao and A. Villanova del Moral, Minimal supersymmetric inverse seesaw: neutrino masses, lepton flavour violation and LHC phenomenology, JHEP 01 (2010) 103 [arXiv:0910.2435] [INSPIRE].

[63] A. Abada, C. Biggio, F. Bonnet, M. Gavela and T. Hambye, Low energy effects of neutrino masses, JHEP 12 (2007) 061 [arXiv:0707.4058] [INSPIRE].

[64] E. Ma, Verifiable radiative seesaw mechanism of neutrino mass and dark matter, Phys. Rev. D 73 (2006) 077301 [hep-ph/0601225] [INSPIRE].

[65] D. Schmidt, T. Schwetz and T. Toma, Direct detection of leptophilic dark matter in a model with radiative neutrino masses, Phys. Rev. D 85 (2012) 073009 [arXiv:1201.0906] [INSPIRE].

[66] R. Bouchand and A. Merle, Running of radiative neutrino masses: the scotogenic model, JHEP 07 (2012) 084 [arXiv: 1205.0008] [INSPIRE].

[67] E. Ma, A. Natale and A. Rashed, Scotogenic $A_{4}$ neutrino model for nonzero $\theta_{13}$ and large $\delta_{C P}$, Int. J. Mod. Phys. A 27 (2012) 1250134 [arXiv:1206.1570] [InSPIRE].

[68] C. Arina, F. Bazzocchi, N. Fornengo, J. Romao and J. Valle, Minimal supergravity sneutrino dark matter and inverse seesaw neutrino masses, Phys. Rev. Lett. 101 (2008) 161802 [arXiv: 0806.3225] [INSPIRE].

[69] H. An, P.B. Dev, Y. Cai and R. Mohapatra, Sneutrino dark matter in gauged inverse seesaw models for neutrinos, Phys. Rev. Lett. 108 (2012) 081806 [arXiv:1110.1366] [INSPIRE].

[70] J.E. Kim, Light pseudoscalars, particle physics and cosmology, Phys. Rept. 150 (1987) 1 [INSPIRE].

[71] N. Arkani-Hamed, D.P. Finkbeiner, T.R. Slatyer and N. Weiner, A theory of dark matter, Phys. Rev. D 79 (2009) 015014 [arXiv:0810.0713] [InSPIRE].

[72] S.Y. Khlebnikov and M. Shaposhnikov, The statistical theory of anomalous fermion number nonconservation, Nucl. Phys. B 308 (1988) 885 [INSPIRE]. 
[73] J.A. Harvey and M.S. Turner, Cosmological baryon and lepton number in the presence of electroweak fermion number violation, Phys. Rev. D 42 (1990) 3344 [INSPIRE].

[74] Y. Burnier, M. Laine and M. Shaposhnikov, Baryon and lepton number violation rates across the electroweak crossover, JCAP 02 (2006) 007 [hep-ph/0511246] [INSPIRE].

[75] M. D'Onofrio, K. Rummukainen and A. Tranberg, The sphaleron rate through the electroweak cross-over, JHEP 08 (2012) 123 [arXiv:1207.0685] [INSPIRE].

[76] S. Shapiro, S. Teukolsky and I. Wasserman, Do neutrino rest masses affect cosmological helium production?, Phys. Rev. Lett. 45 (1980) 669 [INSPIRE]. 